Bull. Nov. Comp. Center, , (2019), 39-52

(C) 2019 NCC Publisher

\title{
State space reduction for time Petri nets with weak semantics
}

\begin{abstract}
Aleksei Zubarev
Abstract. We consider the time Petri nets (an extension of Petri nets), where every transition has its time interval. The policies of time-elapsing and the memory policies define different semantics for time Petri nets. The decidability of many standard problems with an infinite discrete structure depends on the choice of semantics. The state space of the time Petri nets is infinite and non-discrete. It is known that there is a reduction of the state space to discrete one for the time Petri nets with strong semantics. In this paper, we prove that a state space reduction can be applied to weak time Petri nets equipped with intermediate and atomic memory policies.
\end{abstract}

Keywords: State space reduction, weak time Petri net, intermediate memory policy, atomic memory policy.

\section{Introduction}

Petri nets [5] is one of the generally accepted models for the analysis of concurrent and distributed systems. In verification systems, there is an obvious need for considering time. Different timed extensions of Petri nets have been proposed [4][8][3]. They combine the discrete structure and continuous time characteristics.

Time Petri nets are an extension of Petri nets, where every transition has a clock function and a time interval. There are two policies of timeelapsing, which define weak and strong semantics [11]. Time cannot disable a transition in strong semantics, thus the transition has to fire no later than the upper limit of the time interval is reached. On the contrary, all time delays are allowed in the weak semantics. In [2], the authors have proven that the weak semantics and strong semantics are incomparable. It is known that standard verification problems are decidable for the timed extensions of bounded Petri nets with either semantics. However, the choice of semantics plays an important role when we consider models with an unbounded discrete structure. The standard verification problems are undecidable for time Petri nets with the most studied strong semantics.

When a transition $t$ fires, the clocks of some of transitions may be reset. Various ways to reset the clock are considered. The memory policies specify when information about time is kept. The fundamental model of Merlin [4] has an intermediate memory policy. This semantics considers an intermediary marking between consumption tokens from the input places of $t$ and 
production tokens to the output places of $t$. If a transition $t^{\prime}$ is disabled in the intermediary marking, then the clock of $t^{\prime}$ is reset. On the other hand, the atomic semantics considers a firing as one-step. The clock of $t^{\prime}$ is reset only if $t^{\prime}$ is disabled in the marking before $t$ fires. The memory policies for strong semantics have been studied in [1]. In [9], the authors have proven that the marking reachability problem, the coverability problem, and the boundedness problem are decidable for weak time Petri nets with the intermediate semantics. Furthermore, a marking is reachable in such nets if and only if it is reachable in the underlying untimed Petri net. They also show that the atomic semantics is not included in the intermediate one for weak time Petri nets.

The state space of time Petri nets is infinite and non-discrete, which increases the complexity of the model analysis. In [7][6], the transformation to discrete time elapsing was introduced for time Petri nets with strong semantics. This transformation reduces the state space and preserves the properties of the system. In addition, it allows us to use the reachability graph to study a system behavior.

The aim of this paper is to generalize the state space reduction approach [7] for time Petri nets with weak semantics and different memory policies. We prove that any reachable marking of a time Petri net with weak semantics can be obtained by a firing sequence with only integer time elapsing.

The paper is organized as follows. In Section 2, we consider some definitions for Petri nets and time Petri nets. In Section 3, we introduce a parametric firing sequence (a generalization of the firing sequence for time Petri nets) with variable time components. Further, we define an integervalue assignment to variables in the parametric firing sequence. Next, we show that this assignment produces a firing sequence of time Petri nets.

\section{Preliminaries}

\subsection{Time Petri nets}

In this section, some terminology concerning the model of Petri nets with timing constraints (time intervals on the firings of transitions) are defined. We start with recalling the definitions of the structure and behavior of Petri nets (elementary net systems) [10].

\section{Definition 1.}

- A Petri net is a tuple $\mathcal{N}=\left(P, T, F, V, M_{0}\right)$, where $P$ is a finite set of places and $T$ is a finite set of transitions such that $P \cap T=\varnothing$ and $P \cup T \neq \varnothing, F \subseteq(P \times T) \cup(T \times P)$ is a set of arcs (flow relation), $V: F \rightarrow \mathbb{N}$ is a weight of the arcs, $M_{0}: P \rightarrow \mathbb{N}$ is an initial marking such that $M_{0} \not \equiv 0$. For $x \in P \cup T$ let ${ }^{\bullet} x: P \cup T \rightarrow \mathbb{N}$ and $x^{\bullet}: P \cup T \rightarrow \mathbb{N}$ 
be the backward incidence mapping and forward incidence mapping of $x$, respectively.

$$
\cdot x(y)=\left\{\begin{array}{ll}
V(y, x) & \text { if }(y, x) \in F, \\
0 & \text { otherwise. }
\end{array} \quad x^{\bullet}(y)= \begin{cases}V(x, y) & \text { if }(x, y) \in F, \\
0 & \text { otherwise }\end{cases}\right.
$$

- A marking of a Petri net $\mathcal{N}$ is a total function $M: P \rightarrow \mathbb{N}$. A transition $t \in T$ is enabled at a marking $M$ if $t \leqslant M$. Let $\operatorname{En}(M)$ be the set of transitions enabled at $M$. The firing of a transition $t$ enabled at a marking $M$ leads to the new marking $M^{\prime}$ (denoted as $M \stackrel{t}{\longrightarrow} M^{\prime}$ ) iff $M^{\prime}=M-\bullet t+t^{\bullet}$. We write $M \stackrel{\vartheta}{\longrightarrow} M^{\prime}$ iff $\vartheta=t_{1} \ldots t_{k}$ and $M=M^{0}$ $\stackrel{t_{1}}{\longrightarrow} M^{1} \ldots M^{k-1} \stackrel{t_{k}}{\longrightarrow} M^{k}=M^{\prime}$. In this case, $\vartheta$ is a firing sequence of $\mathcal{N}$ from $M$ (to $M^{\prime}$ ), and $M^{\prime}$ is a reachable marking of $\mathcal{N}$ from $M$. Let $R M(\mathcal{N})$ be the set of all reachable markings of $\mathcal{N}$ from $M_{0}$.

Following the approach of [9], we extend the above model to time Petri nets with weak semantics equipped with different memory policies for the firing of transitions.

\section{Definition 2.}

- A time Petri net $(\mathrm{TPN})$ is a pair $\mathcal{T} \mathcal{N}=(\mathcal{N}, D)$, where $\mathcal{N}=\left(P, T, F, V, M_{0}\right)$ is the underlying Petri net and $D: T \rightarrow \mathbb{Q} \geqslant 0 \times(\mathbb{Q} \geqslant 0 \cup\{\infty\})$ is a static timing function associating with each transition a closed interval between two time values. For a transition $t \in T$, the boundaries of the interval $D(t)$ are called the earliest firing time Eft and latest firing time Lft of $t$.

- A state of $\mathcal{T N}$ is a pair $(M, I)$, where $M$ is a marking and $I$ : $E n(M) \longrightarrow \mathbb{R}$ is a dynamic timing function. The initial state of $\mathcal{T N}$ is a pair $S_{0}=\left(M_{0}, I_{0}\right)$, where $M_{0}$ is the initial marking and $I_{0}(t)=0$, for all $t \in \operatorname{En}\left(M_{0}\right)$. A transition $t$ enabled at a marking $M$ can be fired from a state $S=(M, I)$ if $I(t)$ belongs to the interval $D(t)$.

Two types of events are considered for time Petri nets.

(a) The discrete event (firing of a transition) is defined $\forall t \in T$ by

$$
(M, I) \stackrel{t}{\longrightarrow}\left(M^{\prime}, I^{\prime}\right) \text { iff }\left\{\begin{array}{l}
t \in \operatorname{En}(M), \text { and, } \\
M^{\prime}=M-\bullet t+t^{\bullet}, \text { and, } \\
E f t(t) \leqslant I(t) \leqslant L f t(t), \text { and, } \\
\forall t^{\prime} \in \operatorname{En}\left(M^{\prime}\right), I^{\prime}\left(t^{\prime}\right)= \begin{cases}0 & \text { if } \uparrow \text { enabled } \\
I\left(t^{\prime}\right) & \text { otherwise }\end{cases}
\end{array}\right.
$$


(b) The continuous event (the time elapsing) is defined $\forall \tau \in \mathbb{R}_{\geqslant 0}$ by

$$
(M, I) \stackrel{\tau}{\longrightarrow}\left(M, I^{\prime}\right) \text { iff } \forall t^{\prime} \in \operatorname{En}(M), I^{\prime}\left(t^{\prime}\right)=I\left(t^{\prime}\right)+\tau
$$

A predicate $\uparrow$ enabled $_{s}\left(t^{\prime}, M, t\right)$ with $s \in\{I, A\}$ indicates whether we need to reset the clock of $t^{\prime}$ after a firing of a transition $t$ at a marking $M$. Different semantics can be chosen in order to realize these resets. This choice depends on what is called the memory policy [1].

I: Intermediate semantics considers a firing of a transition as two actions: consuming the tokens from input places of $t$, and producing the tokens to output places of $t$. Thus the clocks of $t$ and of the transitions that could not be fired in parallel with $t$ from the marking $M$ are reset.

$\uparrow \operatorname{enabled}_{I}\left(t^{\prime}, M, t\right)=t^{\prime} \in \operatorname{En}\left(M-\bullet t+t^{\bullet}\right) \wedge\left(t^{\prime} \notin E n(M-\bullet t) \vee t=t^{\prime}\right)$

A: Atomic semantics considers a firing of a transition as one action. The clocks of $t$ and of the transitions $t^{\prime} \notin E n(M)$ are reset.

$$
\uparrow \operatorname{enabled}_{A}\left(t^{\prime}, M, t\right)=t^{\prime} \in E n\left(M-\bullet t+t^{\bullet}\right) \wedge\left(t^{\prime} \notin E n(M) \vee t=t^{\prime}\right)
$$

We use the notation $S \stackrel{\sigma}{\longrightarrow} S^{\prime}$ iff $\sigma=\overline{t_{1}} \ldots \overline{t_{k}} \in\left(T \cup \mathbb{R}_{\geqslant 0}\right)^{k}$ and $S=S^{0}$ $\stackrel{\overline{t_{1}}}{\longrightarrow} S^{1} \ldots S^{k-1} \stackrel{\overline{t_{k}}}{\longrightarrow} S^{k}=S^{\prime}(k \geqslant 0)$. In this case, $\sigma$ is a firing sequence of $\mathcal{T N}$ from $S$ (to $S^{\prime}$ ), and $S^{\prime}$ is a reachable state of $\mathcal{T} \mathcal{N}$ from $S$. Let $\mathcal{F S}(\mathcal{T N})$ be the set of all firing sequences of $\mathcal{T N}$ from $S_{0}$, and $R S(\mathcal{T N})$ be the set of all reachable states of $\mathcal{T N}$ from $S_{0}$.

We require the following standard properties for the time elapsing:

- Time determinism: if $S \stackrel{\tau}{\longrightarrow} S^{\prime}$ and $S \stackrel{\tau}{\longrightarrow} S^{\prime \prime}$ with $\tau \in \mathbb{R}_{\geqslant 0}$, then $S^{\prime}=S^{\prime \prime}$.

- 0-delay: $S \stackrel{0}{\longrightarrow} S$

- Additivity: if $S \stackrel{\tau}{\longrightarrow} S^{\prime}$ and $S^{\prime} \stackrel{\tau^{\prime}}{\longrightarrow} S^{\prime \prime}$ with $\tau, \tau^{\prime} \in \mathbb{R}_{\geqslant 0}$, then $S \stackrel{\tau+\tau^{\prime}}{\longrightarrow} S^{\prime \prime}$.

- Continuity: if $S \stackrel{\tau}{\longrightarrow} S^{\prime}$, then for every $\tau^{\prime}, \tau^{\prime \prime} \in \mathbb{R}_{\geqslant 0}$, such that $\tau=$ $\tau^{\prime}+\tau^{\prime \prime}$, there exists an $S^{\prime \prime}$ such that $S \stackrel{\tau^{\prime}}{\longrightarrow} S^{\prime \prime} \stackrel{\tau^{\prime \prime}}{\longrightarrow} S^{\prime}$.

With these properties, a firing sequence can be defined as a finite sequence $\sigma=\tau_{0} t_{1} \tau_{1} \ldots t_{k} \tau_{k}$, where discrete and continuous events alternate. We write $\operatorname{Untimed}(\sigma)=t_{1} \ldots t_{k}$ for the untimed part of $\sigma$ and $\mathcal{U} \mathcal{F} \mathcal{S}(\mathcal{T N})$ for the set of all $\operatorname{Untimed}(\sigma)$, where $\sigma \in \mathcal{F} \mathcal{S}(\mathcal{T N})$. 
Definition 3. Two time Petri nets $\mathcal{T} \mathcal{N}_{1}=\left(\mathcal{N}, D_{1}\right)$ and $\mathcal{T} \mathcal{N}_{2}=\left(\mathcal{N}, D_{2}\right)$ are time equivalent iff there exists a constant $c \neq 0$ such that for each transition $t$ in $\mathcal{N}$ :

- $L f t_{1}(t)=\infty$ iff $L f t_{2}(t)=\infty$,

- $\operatorname{Eft}_{1}(t)=0$ iff $E f t_{2}(t)=0$,

- $L f t_{1}(t)=0$ iff $L f t_{2}(t)=0$,

- $E f t_{1}(t) / E f t_{2}(t)=c$ iff $E f t_{2}(t) \neq 0$,

- $L f t_{1}(t) / L f t_{2}(t)=c$ iff $L f t_{2}(t) \neq 0$.

Theorem 1. Let $\mathcal{T} \mathcal{N}_{1}$ be a time Petri net. Then there exists a time Petri net $\mathcal{T} \mathcal{N}_{2}$ with $D_{2}: T \rightarrow \mathbb{N} \times(\mathbb{N} \cup\{\infty\})$ such that $\mathcal{T} \mathcal{N}_{1}$ and $\mathcal{T} \mathcal{N}_{2}$ are time equivalent.

Ideas of the proof. Compute the L.C.M. $c$ of the denominators of all $E f t_{1}$ and $L f t_{1}$ of $\mathcal{T} \mathcal{N}_{1}$. Then, define $E f t_{2}\left(L f t_{2}\right)$ as the product of $E f t_{1}$ $\left(L f t_{1}\right)$ by $c$.

In the sequel, we will consider the TPN with integer interval bounds.

\section{Integer firing sequence}

The aim of this section is to prove the following: any reachable marking of a time Petri net can be obtained by firing sequence with only integer time elapsing. This generalizes the results of [7] for weak semantics and different clock memory policies.

Definition 4. A state $S=(M, I)$ is called an integer-state if for every $t \in \operatorname{En}(M)$ it holds that $I(t) \in \mathbb{N}$.

Definition 5. A firing sequence $\sigma=\tau_{0} t_{1} \tau_{1} t_{2} \ldots t_{k} \tau_{k}$ is called an integer firing sequence if for every $i=1, \ldots, k$ it holds that $\tau_{i} \in \mathbb{N}$.

Now we define a parametric firing sequence $\omega(x)=x_{0} t_{1} x_{1} \ldots t_{k} x_{k}$ that is a generalization of a firing sequence $\tau_{0} t_{1} \tau_{1} \ldots t_{k} \tau_{k}$, with variables $x_{i}$ instead of a fixed number $\tau_{i}$. In addition, we unite the requirements for values of $x_{i}$ into the set $B_{\omega}$.

Definition 6. Let $\mathcal{T N}=(\mathcal{N}, D)$ be a time Petri net, $\omega=t_{1} \ldots t_{k} \in$ $\mathcal{U} \mathcal{F} \mathcal{S}(\mathcal{T N})$, and $x=\left(x_{0}, \ldots, x_{k}\right)$ be a vector of variables. We define by induction a finite sequence of tuples $\left(\omega_{i}(x), M_{\omega_{i}}, B_{\omega_{i}}, I_{\omega_{i}}\right)$ : 




Figure 1. The time Petri net

Basis: $i=0$,

- $\omega_{0}(x):=x_{0}$

- $M_{\omega_{0}}:=M_{0}$

- $B_{\omega_{0}}:=\varnothing$

- $\forall t \in \operatorname{En}\left(M_{\omega_{0}}\right), I_{\omega_{0}}(t):=x_{0}$

Step: Assume that $\left(\omega_{i-1}(x), M_{\omega_{i-1}}, B_{\omega_{i-1}}, I_{\omega_{i-1}}\right)$ is already defined.

- $\omega_{i}(x):=\omega_{i-1}(x) t_{i} x_{i}$;

- $M_{\omega_{i}}:=M_{\omega_{i-1}}-t_{i}+t_{i}^{\bullet}$

- $B_{\omega_{i}}:=B_{\omega_{i-1}} \cup\left\{E f t\left(t_{i}\right) \leqslant I_{\omega_{i-1}}\left(t_{i}\right) \leqslant L f t\left(t_{i}\right)\right\}$;

- $\forall t \in \operatorname{En}\left(M_{\omega_{i}}\right)$,

$$
I_{\omega_{i}}(t):=\left\{\begin{array}{ll}
x_{i} & \text { if } \uparrow \text { enabled }_{s}\left(t, M_{\omega_{i-1}}, t_{i}\right) \\
I_{\omega_{i-1}}(t)+x_{i} & \text { otherwise }
\end{array} .\right.
$$

Then, the parametric firing sequence $\left(\omega(x), B_{\omega}\right)$ of $\mathcal{T N}$ and the parametric state $\left(S_{\omega}, B_{\omega}\right)$ are defined as follow:

$$
\begin{gathered}
\left(\omega(x), B_{\omega}\right):=\left(\omega_{k}(x), B_{\omega_{k}}\right) \\
\left(S_{\omega}, B_{\omega}\right):=\left(\left(M_{\omega_{k}}, I_{\omega_{k}}\right), B_{\omega_{k}}\right)
\end{gathered}
$$

We remark that $t_{i} \in \operatorname{En}\left(M_{\omega_{i-1}}\right)$ for $i=1 \ldots k$, as there is a firing sequence $\sigma$ such that $\omega=\operatorname{Untimed}(\sigma)$. Then the definition is correct.

Example 1. Let us consider the time Petri net $\mathcal{T N}$ with atomic semantics in Figure 1 and the firing sequence $\sigma=(1.5) t_{1}(0.5) t_{2}(3.2) t_{3}(0.7)$ of $\mathcal{T} \mathcal{N}$. We note that $\sigma$ is not a firing sequence in the case of intermediate semantics, as the clock of $t_{2}$ is reset after $t_{1}$ fires. In strong semantics, the transition $t_{3}$ is dead. For the transition sequence $\omega=\operatorname{Untimed}(\sigma)$ the parametric firing sequence $\left(\omega(x), B_{\omega}\right)$ has the form $\omega(x)=x_{0} t_{1} x_{1} t_{2} x_{2} t_{3} x_{3}$ and 
$B_{\omega}=\left\{\begin{array}{c}E f t\left(t_{1}\right) \leqslant x_{0} \leqslant L f t\left(t_{1}\right), \\ E f t\left(t_{2}\right) \leqslant x_{0}+x_{1} \leqslant L f t\left(t_{2}\right), \\ E f t\left(t_{3}\right) \leqslant x_{2} \leqslant L f t\left(t_{3}\right)\end{array}\right\}=\left\{\begin{array}{cc}x_{0} \geqslant 1, & x_{0} \leqslant 2, \\ x_{0}+x_{1} \geqslant 1, & x_{0}+x_{1} \leqslant 2, \\ x_{2} \geqslant 3, & x_{2} \leqslant 4\end{array}\right\}$.

It is easy to see that the function $\beta(x)=\left(\beta\left(x_{0}\right), \beta\left(x_{1}\right), \beta\left(x_{2}\right), \beta\left(x_{3}\right)\right)=$ $(1,1,3,1)$ is a solution of $B_{\omega}$ and thus $\omega(\beta(x))=(1) t_{1}(1) t_{2}(3) t_{3}(1)$ is the firing sequence of $\mathcal{T N}$. The next Proposition proves this property for any solution of $B_{\omega}$.

We denote the value of a linear function $g(x)$ under the assignment $\beta$ by $[g]_{\beta}$.

Proposition 1. Let $\mathcal{T N}=(\mathcal{N}, D)$ be a time Petri net, $\omega=t_{1} \ldots t_{k} \in$ $\mathcal{U} \mathcal{F} \mathcal{S}(\mathcal{T N})$ and $\left(\omega(x), B_{\omega}\right)=\left(\left(x_{0} t_{1} x_{1} \ldots t_{k} x_{k}\right), B_{\omega}\right)$ be a parametric firing sequence of $\mathcal{T} \mathcal{N}$. Then if $\beta(x)=\left(\beta\left(x_{1}\right), \ldots, \beta\left(x_{k}\right)\right)$ is a solution of $B_{\omega}$, then $\omega(\beta(x))$ is a firing sequence.

Proof. Let us prove that $\left(M_{0}, I_{0}\right) \stackrel{\omega_{i}(\beta(x))}{\longrightarrow}\left(M_{\omega_{i}},\left[I_{\omega_{i}}\right]_{\beta}\right)$ for all $i=0 \ldots k$. Induction on $i$.

Basis: $i=0$. By Definition $6, \omega_{0}(\beta(x))=\beta\left(x_{0}\right) ; M_{\omega_{0}}=M_{0} ; \forall t \in$ $\operatorname{En}\left(M_{\omega_{0}}\right),\left[I_{\omega_{0}}\right]_{\beta}(t)=\beta\left(x_{0}\right)$. Therefore, by Definition $2,\left(M_{0}, I_{0}\right) \stackrel{\omega_{0}(\beta(x))}{\longrightarrow}$ $\left(M_{\omega_{0}},\left[I_{\omega_{0}}\right]_{\beta}\right)$.

Step: Assume that $\left(M_{0}, I_{0}\right) \stackrel{\omega_{i}(\beta(x))}{\longrightarrow}\left(M_{\omega_{i}},\left[I_{\omega_{i}}\right]_{\beta}\right)$ holds for $0 \ldots i-1$, and now prove it for $i$. As $\omega_{i}(x):=\omega_{i-1}(x) t_{i} x_{i}$ we need to show that $\left(M_{\omega_{i-1}},\left[I_{\omega_{i-1}}\right]_{\beta}\right) \stackrel{t_{i} \beta\left(x_{i}\right)}{\longrightarrow}\left(M_{\omega_{i}},\left[I_{\omega_{i}}\right]_{\beta}\right)$. By Definition 6 , we have:

- $t_{i} \in \operatorname{En}\left(M_{\omega_{i-1}}\right)$,

- $M_{\omega_{i}}:=M_{\omega_{i-1}}-{ }^{\circ} t_{i}+t_{i}^{\bullet}$,

- $\operatorname{Eft}\left(t_{i}\right) \leqslant\left[I_{\omega_{i-1}}\right]_{\beta}\left(t_{i}\right) \leqslant L f t\left(t_{i}\right)$ (because $\beta$ is a solution of $B_{\omega_{i}}$ ),

- $\forall t \in \operatorname{En}\left(M_{\omega_{i}}\right),\left[I_{\omega_{i}}\right]_{\beta}(t)-\beta\left(x_{i}\right):=\left\{\begin{array}{ll}0 & \text { if } \uparrow \text { enabled }_{s}\left(t, M_{\omega_{i-1}}, t_{i}\right) \\ {\left[I_{\omega_{i-1}}\right]_{\beta}(t)} & \text { otherwise }\end{array}\right.$.

Then, by Definition 2, we have $\left(M_{\omega_{i-1}},\left[I_{\omega_{i-1}}\right]_{\beta}\right) \stackrel{t_{i}}{\longrightarrow}\left(M_{\omega_{i}},\left[I_{\omega_{i}}\right]_{\beta}-\beta\left(x_{i}\right)\right) \stackrel{\beta\left(x_{i}\right)}{\longrightarrow}$ $\left(M_{\omega_{i}},\left[I_{\omega_{i}}\right]_{\beta}\right)$. Hence $\left(M_{0}, I_{0}\right) \stackrel{\omega(\beta(x))}{\longrightarrow}\left(M_{\omega},\left[I_{\omega}\right]_{\beta}\right)$.

Now we want to study the structure of the inequalities in the set $B_{\omega}$ and the structure of the function $I_{\omega}$. 
Proposition 2. Let $\mathcal{T N}=(\mathcal{N}, D)$ be a time Petri net, $\omega=t_{1}, \ldots, t_{k} \in$ $\mathcal{U F S}(\mathcal{T N})$ and $\left(\omega_{i}(x), M_{\omega_{i}}, B_{\omega_{i}}, I_{\omega_{i}}\right)$ be sequences of tuples from Definition 6. Then it holds that

(a) If $t \in \operatorname{En}\left(M_{\omega_{i}}\right)$ then the variable $x_{i}$ appears in $I_{\omega_{i}}(t)$.

(b) If $t \in \operatorname{En}\left(M_{\omega_{i}}\right)$ and the variable $x_{n}$ appears in $I_{\omega_{i}}(t)$ then $n \leqslant i$ and every variable $x_{l}$ with $n \leqslant l \leqslant i$ also appears in $I_{\omega_{i}}(t)$.

(c) If $t \in \operatorname{En}\left(M_{\omega_{i}}\right)$ and the variables $x_{m}$ and $x_{n}$ with $m \leqslant n$ appear in $I_{\omega_{i}}(t)$ then every variable $x_{l}$ with $m \leqslant l \leqslant n$ also appears in $I_{\omega_{i}}(t)$.

(d) If $t_{1}, t_{2} \in \operatorname{En}\left(M_{\omega_{i}}\right)$ then either each variable appearing in $I_{\omega_{i}}\left(t_{1}\right)$ also appears in $I_{\omega_{i}}\left(t_{2}\right)$ or vice versa.

(e) If $g(x) \leqslant r$ is an inequality in $B_{\omega_{i}}$ and the variables $x_{m}$ and $x_{n}$ with $m \leqslant n$ appear in $g(x)$ then every variable $x_{l}$ with $m \leqslant l \leqslant n$ also appears in $g(x)$.

Proof. Induction on $i$.

Basis: For $i=0$, all five assertions follow immediately from Definition 6 .

Step: We assume that the assertions hold for $0 \ldots i-1$, and now prove them for $i$.

(a) The assertion follows from the definition of $I_{\omega_{i}}$.

(b) By Definition 6, either $I_{\omega_{i}}(t)=x_{i}$ or $I_{\omega_{i}}(t)=I_{\omega_{i-1}}(t)+x_{i}$. Thus, the result follows from the induction hypothesis.

(c) The assertion follows from (b).

(d) According to (b), $I_{\omega_{i}}\left(t_{1}\right)$ and $I_{\omega_{i}}\left(t_{2}\right)$ have the form:

$$
\begin{gathered}
I_{\omega_{i}}\left(t_{1}\right)=x_{i-n}+x_{i-(n-1)}+\ldots+x_{i} \\
I_{\omega_{i}}\left(t_{2}\right)=x_{i-m}+x_{i-(m-1)}+\ldots+x_{i}
\end{gathered}
$$

If $n \leqslant m$ then all variables appearing in $I_{\omega_{i}}\left(t_{1}\right)$ also appear in $I_{\omega_{i}}\left(t_{2}\right)$. If $m \leqslant n$ then all variables appearing in $I_{\omega_{i}}\left(t_{2}\right)$ also appear in $I_{\omega_{i}}\left(t_{1}\right)$.

(e) Since $g(x)=I_{\omega_{n}}(t)$ for some $n \in\{0,1, \ldots, i-1\}$, the result follows from (c).

By Definition 6 , the set $B_{\omega}$ has the form $\left\{g_{i}(x) \leqslant c_{i}, h_{i}(x) \geqslant r_{i} \mid i=\right.$ $1 \ldots k\}$. Denote by $\widetilde{B}_{\omega}=\left\{g_{i}(x), h_{i}(x) \mid i=1 \ldots k\right\}$ the set of variable parts of inequalities from $B_{\omega}$. We further provide the construction of an integervalue assignment $\beta_{\omega}$ to variables in the parametric firing sequence. 
Definition 7. Let $\mathcal{T N}=(\mathcal{N}, D)$ be a time Petri net, $\sigma=\tau_{0} t_{1} \tau_{1} \ldots t_{k} \tau_{k} \in$ $\mathcal{F S}(\mathcal{T N})$ be a firing sequence, $\omega=\operatorname{Untimed}(\sigma)$, and $\left(\left(x_{0} t_{1} x_{1} \ldots t_{k} x_{k}\right), B_{\omega}\right)$ be a parametric firing sequence of $\mathcal{T} \mathcal{N}$. Define by induction a finite sequence of assignments $\beta_{i}:\left\{x_{0}, \ldots, x_{k}\right\} \rightarrow \mathbb{R}_{\geqslant 0}$ :

Basis: $\beta_{0}\left(x_{j}\right):=\tau_{j}$ for all $j=0,1, \ldots, k$.

Step: Assume that $\beta_{i-1}$ is already defined. In the construction of $\beta_{i}$ we use the following function:

$$
\underline{\beta_{i}}:=\left\{\begin{array}{ll}
\beta_{i-1}(x) & \text { if } x \neq x_{k-(i-1)} \\
\left\lfloor\beta_{i-1}(x)\right\rfloor & \text { otherwise }
\end{array} .\right.
$$

We now define $\beta_{i}$ by

$$
\beta_{i}(x):=\left\{\begin{array}{ll}
\beta_{i-1}(x) & \text { if } x \neq x_{k-(i-1)} \\
\left\lfloor\beta_{i-1}(x)\right\rfloor & \text { if } x=x_{k-(i-1)} \text { and } \\
& \forall g \in \widetilde{B}_{\omega} \rightarrow\left\lfloor[g]_{\beta_{0}}\right\rfloor-1<[g]_{\underline{\beta_{i}}} \\
\left\lceil\beta_{i-1}(x)\right\rceil & \text { otherwise }
\end{array} .\right.
$$

We put $\beta_{\omega}\left(x_{j}\right):=\beta_{k+1}\left(x_{j}\right)$ for all $j=0,1, \ldots, k$.

Obviously, the values of this function are all integer. Clearly, an assignment $\beta_{i}$ is obtained from $\beta_{i-1}$ by rounding the value $\beta_{i-1}\left(x_{k-(i-1)}\right)$. The next proposition now follows immediately.

Proposition 3. For all $i \in\{0,1, \ldots, k+1\}$ it holds that

(a) $\beta_{i}\left(x_{j}\right)=\beta_{0}\left(x_{j}\right)$ for $j \in\{0,1, \ldots, k-i\}$

(b) $\beta_{i}\left(x_{j}\right)=\beta_{k+1}\left(x_{j}\right)$ for $j \in\{k-(i-1), k-(i-2), \ldots, k\}$

Example 2. Let us again consider the time Petri net $\mathcal{T N}$ with atomic semantics in Figure 1. It is clear that $\widetilde{B}_{\omega}=\left\{x_{0}, x_{0}+x_{1}, x_{2}\right\}$ and $\left(\left\lfloor\left[x_{0}\right]_{\beta_{0}}\right\rfloor-\right.$ $\left.1,\left\lfloor\left[x_{0}+x_{1}\right]_{\beta_{0}}\right\rfloor-1,\left\lfloor\left[x_{2}\right]_{\beta_{0}}\right\rfloor-1\right)=(0,1,2)$. In the table in Figure 2 we produce integer values for variables $\left\{x_{0}, x_{1}, x_{2}, x_{3}\right\}$.

Thus, we obtain the integer firing sequence $\omega\left(\beta_{\omega}(x)\right)=(2) t_{1}(0) t_{2}(3) t_{3}(0)$. We will show that if $\omega \in \mathcal{U} \mathcal{F S}(\mathcal{T} \mathcal{N})$ then $\omega\left(\beta_{\omega}(x)\right)$ is an integer firing sequence.

Consider some properties for the functions $\beta_{i}$.

Lemma 1. For all $i \in\{0,1, \ldots, k+1\}$ it holds that:

(a) $\forall g\left(g \in \widetilde{B}_{\omega} \rightarrow[g]_{\beta_{i}} \in\left(\left\lfloor[g]_{\beta_{0}}\right\rfloor-1,\left\lceil[g]_{\beta_{0}}\right]+1\right)\right)$,

(b) $\forall t\left(t \in \operatorname{En}\left(M_{\omega}\right) \rightarrow\left[I_{\omega}(t)\right]_{\beta_{i}} \leqslant\left[I_{\omega}(t)\right]_{\beta_{0}}\right)$,

(c) $\left[\sum_{j=0}^{k} x_{j}\right]_{\beta_{i}} \leqslant\left[\sum_{j=0}^{k} x_{j}\right]_{\beta_{0}}$ 


\begin{tabular}{|c||c|c|c|c|c|}
\hline & $i=0$ & $i=1$ & $i=2$ & $i=3$ & $i=4$ \\
\hline$\underline{\beta_{i}}\left(x_{0}\right)$ & - & 1.5 & 1.5 & 1.5 & 1 \\
$\underline{\beta_{i}}\left(x_{1}\right)$ & - & 0.5 & 0.5 & 0 & 0 \\
$\underline{\beta_{i}}\left(x_{2}\right)$ & - & 3.2 & 3 & 3 & 3 \\
$\underline{\beta_{i}}\left(x_{3}\right)$ & - & 0 & 0 & 0 & 0 \\
\hline$\left[x_{0}\right]_{\beta_{i}}$ & - & 1.5 & 1.5 & 1.5 & 1 \\
{$\left[x_{0}+x_{1}\right]_{\underline{\beta_{i}}}$} & - & 2 & 2 & 1.5 & 1 \\
{$\left[x_{2}\right]_{\underline{\beta}_{i}}$} & - & 3.2 & 3 & 3 & 3 \\
\hline$\left\lfloor[g]_{\beta_{0}}\right]-1<[g]_{\beta_{i}}$ & - & true & true & true & false \\
\hline$\beta_{i}\left(x_{0}\right)$ & 1.5 & 1.5 & 1.5 & 1.5 & 2 \\
$\beta_{i}\left(x_{1}\right)$ & 0.5 & 0.5 & 0.5 & 0 & 0 \\
$\beta_{i}\left(x_{2}\right)$ & 3.2 & 3.2 & 3 & 3 & 3 \\
$\beta_{i}\left(x_{3}\right)$ & 0.7 & 0 & 0 & 0 & 0 \\
\hline
\end{tabular}

Figure 2. The construction of the assignment $\beta_{\omega}$

Proof. Induction on $i$.

Basis: $i=0$. For $i=0$, there is nothing to prove.

Step: We assume that the assertions (a), (b) and (c) hold for $0, \ldots, i$ and now prove them for $i+1$.

If $\beta_{i}\left(x_{k-i}\right) \in \mathbb{N}$, then $\beta_{i+1}=\beta_{i}$ and thus all assertions follow immediately from the induction hypothesis.

Therefore, we assume that $\beta_{i}\left(x_{k-i}\right)$ is not an integer. There are only two possible values that $\beta_{i+1}\left(x_{k-i}\right)$ can take:

Case 1: $\beta_{i+1}\left(x_{k-i}\right)=\left\lfloor\beta_{i}\left(x_{k-i}\right)\right\rfloor$ Hence, it holds that

$$
\beta_{i+1}(x) \leqslant \beta_{i}(x) \text { for all } x \in\left\{x_{0}, \ldots, x_{k}\right\} .
$$

(a): Let $g \in \widetilde{B}_{\omega}$.

Using (1), we get $[g]_{\beta i+1} \leqslant[g]_{\beta i}$. Hence by this and by the induction hypothesis, $[g]_{\beta i+1} \leqslant[g]_{\beta i}<\left[[g]_{\beta_{0}}\right]+1$.

As $\beta_{i+1}\left(x_{k-i}\right)=\left\lfloor\beta_{i}\left(x_{k-i}\right)\right\rfloor$, the corresponding criterion

$$
\forall h \in \widetilde{B}_{\omega} \rightarrow\left\lfloor[h]_{\beta_{0}}\right\rfloor-1<[h]_{\underline{\beta_{i+1}}} .
$$

is fulfilled. Since $\underline{\beta_{i+1}}=\beta_{i+1}$, we have $\left\lfloor[g]_{\beta_{0}}\right\rfloor-1<[g]_{{\underline{\beta_{i+1}}}}=[g]_{\beta_{i+1}}$. 
(b): Let $t \in \operatorname{En}\left(M_{\omega}\right)$. Using (1), we get $\left[I_{\omega}(t)\right]_{\beta_{i+1}} \leqslant\left[I_{\omega}(t)\right]_{\beta_{i}}$. Hence by this and by the induction hypothesis, $\left[I_{\omega}(t)\right]_{\beta_{i+1}} \leqslant\left[I_{\omega}(t)\right]_{\beta_{i}} \leqslant$ $\left[I_{\omega}(t)\right]_{\beta_{0}}+1$.

(c): Using (1), we get $\left[\sum_{j=0}^{k} x_{j}\right]_{\beta_{i+1}} \leqslant\left[\sum_{j=0}^{k} x_{j}\right]_{\beta_{i}}$. Hence by this and by the induction hypothesis, $\left[\sum_{j=0}^{k} x_{j}\right]_{\beta_{i+1}} \leqslant\left[\sum_{j=0}^{k} x_{j}\right]_{\beta_{i}} \leqslant\left[\sum_{j=0}^{k} x_{j}\right]_{\beta_{0}}+1$.

Case 2: $\beta_{i+1}\left(x_{k-i}\right)=\left\lceil\beta_{i}\left(x_{k-i}\right)\right\rceil$. Hence, it holds that

$$
\beta_{i}(x) \leqslant \beta_{i+1}(x) \text { for all } x \in\left\{x_{0}, \ldots, x_{k}\right\} .
$$

Moreover, $\widetilde{B}_{\omega}$ contains $h$ such that

$$
[h]_{\underline{\beta_{i+1}}} \leqslant\left\lfloor[h]_{\beta_{0}}\right\rfloor-1 .
$$

and $x_{k-i}$ appears in $h$. Thus, we have $[h]_{\beta_{i+1}}=[h]_{\beta_{i}}-\beta_{i}\left(x_{k-i}\right)+\beta_{i+1}\left(x_{k-i}\right)=$ $[h]_{\beta_{i}}-\beta_{i}\left(x_{k-i}\right)+\left[\beta_{i}\left(x_{k-i}\right)\right]=[h]_{\beta_{i}}-\beta_{i}\left(x_{k-i}\right)+\left\lfloor\beta_{i}\left(x_{k-i}\right)\right\rfloor+1=[h]_{\beta_{i+1}}+1 \leqslant$ $\left\lfloor[h]_{\beta_{0}}\right\rfloor$, hence

$$
[h]_{\beta_{i+1}} \leqslant\left\lfloor[h]_{\beta_{0}}\right\rfloor \leqslant[h]_{\beta_{0}}
$$

Let $m$ and $n$ be the minimal and maximal variable indices appearing in $h$, respectively. From Proposition 2(e) it follows that

$$
h=\underbrace{x_{m}+\ldots+x_{k-(i+1)}}_{h_{1}}+\underbrace{x_{k-i}+\ldots+x_{n}}_{h_{2}} .
$$

(a): Suppose to the contrary that there exists $g \in \widetilde{B}$ such that $[g]_{\beta_{i+1}} \geqslant$ $\left\lceil[g]_{\beta_{0}}\right]+1$ Then $x_{k-i}$ appears in $g$ and

$$
[g]_{\beta_{i+1}} \geqslant\left[[g]_{\beta_{0}}\right]+1 \geqslant[g]_{\beta_{0}}+1 .
$$

Let $m^{\prime}$ and $n^{\prime}$ be the minimal and maximal variable indices appearing in $g$, respectively. From Proposition 2(e) it follows that

$$
g=\underbrace{x_{m^{\prime}}+\ldots+x_{k-(i+1)}}_{g_{1}}+\underbrace{x_{k-i}+\ldots+x_{n^{\prime}}}_{g_{2}} .
$$

We now consider the relationship between the two maximal indices $n$ and $n^{\prime}$. There are three possibilities:

- $n=n^{\prime}$. Then $h_{2}=g_{2}$. According to Proposition 3(a), $\left[h_{1}\right]_{\beta_{i+1}}=$ $\left[h_{1}\right]_{\beta_{0}}$ and $\left[g_{1}\right]_{\beta_{i+1}}=\left[g_{1}\right]_{\beta_{0}}$. Thus, by (6) we have $\left[g_{2}\right]_{\beta_{i+1}}-$ $\left[g_{2}\right]_{\beta_{0}} \geqslant 1$. But by (4) $\left[g_{2}\right]_{\beta_{i+1}}-\left[g_{2}\right]_{\beta_{0}} \leqslant 0$ and we reach a contradiction.

- $n<n^{\prime}$. Then 


$$
g=g_{1}+h_{2}+\underbrace{x_{n+1}+\ldots+x_{n^{\prime}}}_{g_{3}} .
$$

According to Proposition 3, $\left[g_{1}+h_{2}\right]_{\beta_{k-n}}=\left[g_{1}+h_{2}\right]_{\beta_{0}}$ and $\left[g_{3}\right]_{\beta_{k-n}}=\left[g_{3}\right]_{\beta_{i+1}}=\left[g_{3}\right]_{\beta_{k+1}}$. Thus, by (4) we have $[g]_{\beta_{i+1}} \leqslant$ $[g]_{\beta_{k-n}}$. Since $k-i \leqslant n$, it follows that $k-n<i+1$. By $(6)$, $[g]_{\beta_{i+1}} \geqslant\left[[g]_{\beta_{0}}\right]+1$. As a result, we obtain $[g]_{\beta_{k-n}} \geqslant\left[[g]_{\beta_{0}}\right]+1$, which contradicts the induction assumption.

- $n^{\prime}>n$. Then

$$
h=h_{1}+g_{2}+\underbrace{x_{n^{\prime}+1}+\ldots+x_{n}}_{h_{3}} .
$$

According to Proposition 3, $\left[h_{1}+g_{2}\right]_{\beta_{k-n^{\prime}}}=\left[h_{1}+g_{2}\right]_{\beta_{0}}$ and $\left[h_{3}\right]_{\beta_{k-n^{\prime}}}=\left[h_{3}\right]_{\beta_{i+1}}=\left[h_{3}\right]_{\beta_{k+1}}$. Thus, by $(6)$ we have $[h]_{\beta_{i+1}}-$ $[h]_{\beta_{k-n^{\prime}}} \geqslant 1$. Since $k-i \leqslant n^{\prime}$, it follows that $k-n^{\prime}<i+1$. By $(4),[h]_{\beta_{i+1}}-1 \leqslant\left\lfloor[h]_{\beta_{0}}\right\rfloor-1$. As a result, we obtain $[h]_{\beta_{k-n^{\prime}}} \leqslant$ $\left[[h]_{\beta_{0}}\right\rfloor-1$, which contradicts the induction assumption.

(b): Let $t \in \operatorname{En}\left(M_{\omega}\right)$. If $x_{k-i}$ does not appear in $I_{\omega}(t)$, then $\left[I_{\omega}(t)\right]_{\beta_{i+1}}=$ $\left[I_{\omega}(t)\right]_{\beta_{i}}$, and $\left[I_{\omega}(t)\right]_{\beta_{i+1}} \leqslant\left[I_{\omega}(t)\right]_{\beta_{0}}$ follows from the induction hypothesis. Suppose that $x_{k-i}$ appear in $I_{\omega}(t)$. Let $\tilde{n}$ be the minimal variable index appearing in $I_{\omega}(t)$, respectively. From Proposition 2(b) it follows that

$$
I_{\omega}(t)=\underbrace{x_{\widetilde{n}}+\ldots+x_{k-(i+1)}}_{I_{1}}+\underbrace{x_{k-i}+\ldots+x_{n}}_{h_{2}}+\underbrace{x_{n+1}+\ldots+x_{k}}_{I_{2}} .
$$

According to Proposition 3, $\left[h_{2}\right]_{\beta_{k-n}}=\left[h_{2}\right]_{\beta_{0}},\left[I_{1}\right]_{\beta_{k-n}}=\left[I_{1}\right]_{\beta_{i+1}}$, and $\left[I_{2}\right]_{\beta_{k-n}}=\left[I_{2}\right]_{\beta_{i+1}}$. Thus, by (4) we have $\left[I_{\omega}(t)\right]_{\beta_{i+1}} \leqslant\left[I_{\omega}(t)\right]_{\beta_{k-n}}$. Since $k-n<i+1$, then by the induction assumption, $\left[I_{\omega}(t)\right]_{\beta_{i+1}} \leqslant$ $\left[I_{\omega}(t)\right]_{\beta_{0}}$.

(c):

$$
\sum_{j=0}^{k} x_{j}=\underbrace{x_{0}+\ldots+x_{k-(i+1)}}_{\Sigma_{1}}+\underbrace{x_{k-i}+\ldots+x_{n}}_{h_{2}}+\underbrace{x_{n+1}+\ldots+x_{k}}_{\Sigma_{2}} .
$$

According to Proposition 3, $\left[h_{2}\right]_{\beta_{k-n}}=\left[h_{2}\right]_{\beta_{0}},\left[\Sigma_{1}\right]_{\beta_{k-n}}=\left[\Sigma_{1}\right]_{\beta_{i+1}}$, and $\left[\Sigma_{2}\right]_{\beta_{k-n}}=\left[\Sigma_{2}\right]_{\beta_{i+1}}$. Thus, by (4) we have $\left[\sum_{j=0}^{k} x_{j}\right]_{\beta_{i+1}} \leqslant\left[\sum_{j=0}^{k} x_{j}\right]_{\beta_{k-n}}$. 


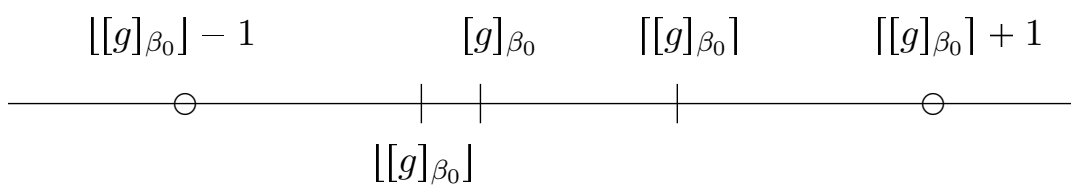

Figure 3. An inequality $g(x) \in B_{\omega}$

Since $k-n<i+1$, then by the induction assumption, $\left[\sum_{j=0}^{k} x_{j}\right]_{\beta_{i+1}} \leqslant$ $\left[\sum_{j=0}^{k} x_{j}\right]_{\beta_{0}}$

Corollary 1. $\beta_{\omega}(x)$ is a solution of $B_{\omega}$.

Consider an arbitrary $(g(x) \leqslant c) \in B_{\omega}$ in Figure 3. According to Lemma 1 , we have $\left.[g]_{\beta_{\omega}} \in\left(\left\lfloor[g]_{\beta_{0}}\right\rfloor-1,\left[[g]_{\beta_{0}}\right\rceil+1\right)\right)$. Since $[g]_{\beta_{0}} \leqslant c$ and $c \in \mathbb{N}$, it follows that $[g]_{\beta_{\omega}} \leqslant c$. Similarly, if $(h(x) \geqslant r) \in B_{\omega}$, then $[h]_{\beta_{\omega}} \geqslant r$. Thus $\beta_{\omega}(x)$ is a solution of $B_{\omega}$.

If we combine Corollary 1, Proposition 1, and Lemma 1, we get the following theorem:

Theorem 2. Let $\mathcal{T} \mathcal{N}=(\mathcal{N}, D)$ be a time Petri net, $\sigma=\tau_{0} t_{1} \tau_{1} \ldots t_{k} \tau_{k}$ be a firing sequence of $\mathcal{T} \mathcal{N},\left(M_{0}, I_{0}\right) \stackrel{\sigma}{\longrightarrow}(M, I), \omega=\operatorname{Untimed}(\sigma),\left(\omega(x), B_{\omega}\right)=$ $\left(\left(x_{0} t_{1} x_{1} \ldots t_{k} x_{k}\right), B_{\omega}\right)$ be a parametric firing sequence, $\left(S_{\omega}, B_{\omega}\right)=\left(\left(M_{\omega}, I_{\omega}\right), B_{\omega}\right)$ be a parametric state. Then there exists an assignment $\beta_{\omega}:\left\{x_{0}, x_{1}, \ldots, x_{k}\right\} \rightarrow$ $\mathbb{N}$ for which the following holds:

- $\omega\left(\beta_{\omega}(x)\right)$ is a firing sequence of $\mathcal{T} \mathcal{N}$,

- $\forall t \in \operatorname{En}\left(M_{\omega}\right)$ it holds that $\left[I_{\omega}(t)\right]_{\beta_{\omega}} \leqslant I(t)$,

- $\left[\sum_{j=0}^{k} x_{j}\right]_{\beta_{\omega}} \leqslant \sum_{j=0}^{k} \tau_{j}$.

As the examples show, the assignment $\beta_{\omega}$ described in the theorem is not uniquely determined. By the theorem, we get one of the most important properties. 
Corollary 2. Let $M$ be a marking of an arbitrary reachable state in a TPN $\mathcal{T N}$. Then $M$ is a marking of a reachable integer-state in $\mathcal{T N}$ with an integer firing sequence.

\section{Conclusion}

In this paper, we have studied the state space reduction for time Petri nets with weak semantics. We have shown that any reachable marking of a weak time Petri net with intermediate and atomic memory policies can be obtained by an integer firing sequence. In the future, this allows us to consider only time Petri nets with integer time elapsing and integer bounds of time intervals. Therefore, we can use only the time elapsing of length 1 .

Further we plan to introduce time processes to represent the causal behavior of time Petri nets with weak semantics equipped with different memory policies. The casual semantics will be required to develop equivalence with partial order semantics for weak time Petri nets. 


\section{References}

[1] Berard B. et al. Comparison of different semantics for time Petri nets // Proc. of International Symposium on Automated Technology for Verification and Analysis. - Springer, Berlin, Heidelberg. - 2005. - P. 293 - 307.

[2] Boyer M., Roux O. H. On the compared expressiveness of arc, place and transition time Petri nets // Fundamenta Informaticae. - 2008. - Vol. 88, No. 3. P. $225-249$.

[3] Hanisch H. M. Analysis of place/transition nets with timed arcs and its application to batch process control // Proc. of International Conference on Application and Theory of Petri Nets. - Springer, Berlin, Heidelberg. - 1993. - P. $282-299$.

[4] Merlin P. M. A Study of the Recoverability of Computing Systems // PhD thesis - Irvine: Univ. California. - 1974.

[5] Petri C. A. Kommunikation mit automation // Dissertation: Univ. Bonn. 1962.

[6] Popova L. On time Petri nets // Information Processing and Cybernetics, EIK. - 1991. - Vol. 27, No. 4. - P. 227-244.

[7] Popova-Zeugmann L. Time Petri nets // Time and Petri Nets. - Springer, Berlin, Heidelberg. - 2013. - P. 31-137.

[8] Ramchandani C. Analysis of asynchronous concurrent systems by timed Petri nets // Dissertation: Massachusetts Institute of Technology. - 1973.

[9] Reynier P. A., Sangnier A. Weak time Petri nets strike back! // International Conference on Concurrency Theory. - Springer, Berlin, Heidelberg, 2009. P. 557-571.

[10] Rozenberg G., Engelfriet J. Elementary net systems // Advanced Course on Petri Nets. - Springer, Berlin, Heidelberg. - 1996. - P. 12-121.

[11] Sifakis J., Yovine S. Compositional specification of timed systems // Annual Symposium on Theoretical Aspects of Computer Science. - Springer, Berlin, Heidelberg. - 1996. - P. 345-359. 